\title{
Aneurysmal bone cyst in the inferior nasal turbinate in a pediatric patient: A case report
}

\author{
Sara Torretta ${ }^{1,2}$, Daniele Di Pasquale ${ }^{1}$, Daniela Carioli ${ }^{1}$, Claudio Guastella ${ }^{1}$, Tullio Ibba ${ }^{1}$, \\ Paola Marchision ${ }^{1,3}$, Lorenzo Pignataro ${ }^{1,2}$ \\ ${ }^{1}$ Fondazione IRCSS Ca' Granda Ospedale Maggiore Policlinica, ${ }^{2}$ Depatment of Clinical Sciences and Community Healthy, \\ and ${ }^{3}$ Department of Pathophysiology and Transplantation, University of Milan, Milan, Italy.E-mail: sara.torretta@unimi.it \\ Received: 4th October 2017, Received: 16th November 2017, Accepted: 27th November 2017
}

SUMMARY: Torretta S, Di Pasquale D, Carioli D, Guastella C, Ibba T, Marchision $\mathrm{P}$, Pignataro L. Aneurysmal bone cyst in the inferior nasal turbinate in a pediatric patient: A case report. Turk J Pediatr 2018; 60: 747-750.

Aneurysmal bone cysts (ABCs) are non-neoplastic, highly vascularised bone lesions that rarely involve the craniofacial complex. We describe the first case of an $\mathrm{ABC}$ involving the inferior turbinate in a 17-month-old boy who attended our Pediatric Emergency Department because of recurrent epistaxis, and discuss the diagnostic and therapeutic work-up in children with nasal masses.

Key words: aneurysmal bone cyst, pediatric tumour, inferior turbinate, nasal bleeding.

An aneurysmal bone cyst $(\mathrm{ABC})$ is a nonneoplastic, benign, locally expanded osteolytic and highly vascularised bone lesion. It was first described as a humerus ossifying hematoma by Van Arsdale ${ }^{1}$ in 1893, but subsequently Jaffe and Lichtenstein ${ }^{2}$ gave it its current name. Although any bony site may be involved, long bone metaphyses (mainly the tibia and femur) are the most frequent location, followed by the spinal cord (14\%), the upper extremities (10\%), and the pelvis $(9 \%) .^{3}$ The craniofacial complex is affected in about $1-3 \%$ of cases ${ }^{4,5}$; the most frequently involved site is the mandible, but there have been rare reports of $A B C s$ of the orbit, lacrimal bone, and paranasal sinuses, including the sphenoid and the ethmoid. ${ }^{6-8}$

$A B C s$ mainly occur during the first and second decades of life, with no significant difference in incidence between sexes. ${ }^{3}$ They are classified as primary when they are not associated with another condition, and secondary when they occur in patients with fibrous dysplasia, giant cell tumours or osteoblastomas (2030\%). ${ }^{3}$ Previous studies have described their indolent and asymptomatic presentation, and the painful and invasive lesions that subsequently impair neurological function. ${ }^{9}$ The most frequent findings in the case of mandible
ABCs are swelling, dull facial pain and loose teeth, whereas those of sinonasal ABCs are unilateral rhinorrhea with or without nasal obstruction, epiphora, proptosis, and a loss of visual acuity. ${ }^{10-12}$

We here describe the first published case of an $A B C$ located in the inferior nasal turbinate of a pediatric patient.

\section{Case Report}

A 17-month-old boy was referred to our Pediatric Emergency Department after three days of recurrent and self-limiting right nostril epistaxis with ispilateral nasal wall swelling. His parents said he had been affected by persistent nasal obstruction for four months, and that he had undergone repeated topical antibiotic therapy for recurrent episodes of right conjunctivitis and epiphora.

Upon physical examination, the patient presented with right epiphora, slight hyperemia of the right inferior eyelid without swelling, proptosis or impaired ocular motility, and swelling of the ipsilateral nasal wall. Anterior rhinoscopy and flexible nasopharyngeal fibre endoscopy showed that the right nasal cavity was completely obstructed by a reddish, thick and easily bleeding mass covered by mucous 
secretions, and magnetic resonance imaging (MRI) revealed an expansive, multilocular and vascularized mass occupying the whole of the right nasal cavity without any connection to intracranial space (Fig. 1). The mass diverted the cartilaginous nasal septum toward the left nasal cavity, blocked the right nasolacrimal duct, and thinned the medial wall of the right maxillary sinus. The ipsilateral middle and inferior nasal turbinates were no longer recognizable, and reactive inflammation of the right ethmoid was also observed. Computed tomography (CT) confirmed the MRI findings (Fig. 2.), and suggested an $\mathrm{ABC}$ as the possible etiology.

Suspecting a vascular lesion, we used transfemoral angiography to inspect the maxillofacial region but did not find any vascular anomaly. Consequently, after evacuating approximately $2 \mathrm{~mL}$ of bloody material from the mass, the mass itself was surgically removed under endoscopic guidance with the patient under general anesthesia. As the lesion was found to originate from the infero-lateral aspect of the right inferior turbinate (which was dislocated upwards and medially towards the middle meatus) and the nasal septum, which was contralaterally diverted, the lesion and the right inferior turbinate were resected in a piecemeal manner. Right nasal packing was used to control the minor residual bleeding, and was removed the day after.

A histopathological examination revealed bony tissue fragments with a number of vascular and dilated structures associated with multinucleated giant cells, thus confirming the radiological suspicion of $\mathrm{ABC}$ (Fig. 3). The post-operative course was uneventful and the patient was discharged two days after surgery. There were no recurrences during the 12-month follow-up.

Informed consent for the procedures and the publication of this case report was obtained from the child's parents.

\section{Discussion}

The precise etiology of ABCs is unknown, but the most widely accepted pathophysiological theory ${ }^{2,3}$ suggests an initial bone trauma leading to histological modifications such as the creation of an intra-osseous hematoma or arteriovenous shunt and malformations: an

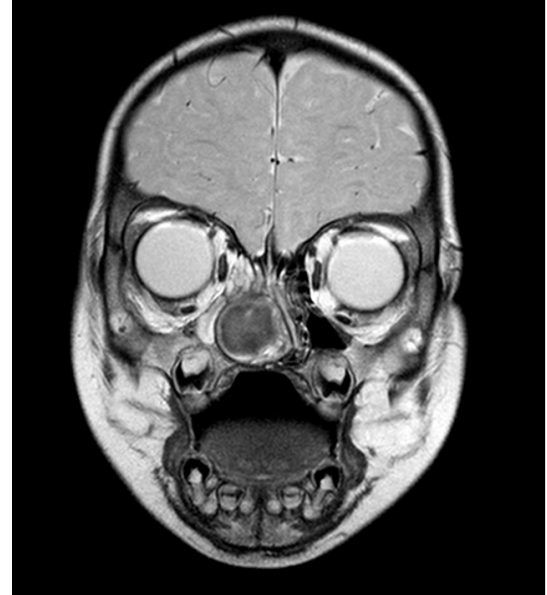

Fig. 1. A T2 MRI scan showing a multilocular cystic mass with blood-fluid levels occupying the right nasal cavity and dislocating the nasal septum.

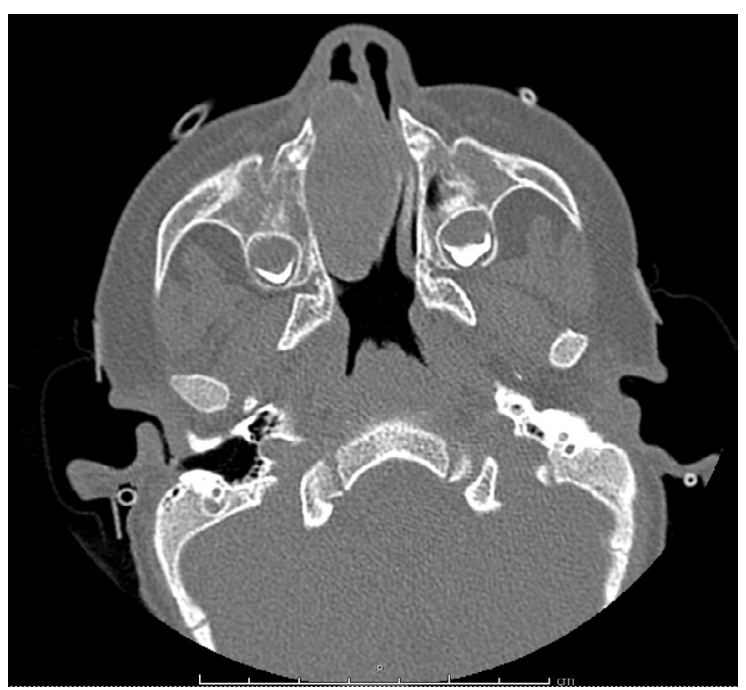

Fig. 2. A CT scan confirmed the MRI findings, and showed the local osteolytic activity of the mass.

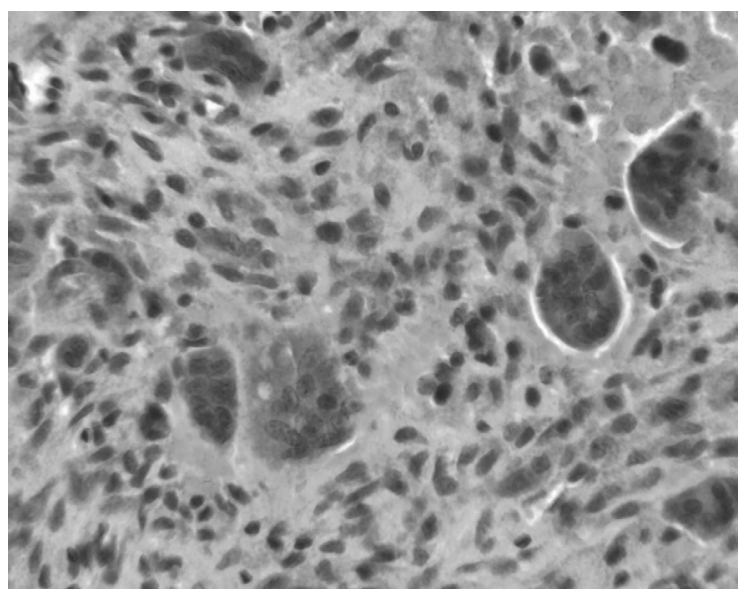

Fig. 3. Histopathological assessment revealed bony tissue fragments with a number of vascular and dilated structures associated with multinucleated giant cells. 
arteriovenous shunt would increase pressure in intra-osseous vessels, thus leading to the expansion of the vascular tissue bed, subsequent bone resorption, and the formation of a cystic cavity. Chromosomal alterations in $17 p$ and $16 q$ sequences have also been recently detected in patients with ABCs. ${ }^{13}$

The clinical features of ABCs depend on their anatomical location. In patients with ethmoidal $\mathrm{ABCs}$, the most frequently reported clinical manifestations include diplopia, proptosis, visual loss or blurry vision, nasolacrimal duct obstruction with epiphora and recurrent congiuntivitis, nasal obstruction, rhinorrhea, and anosmia. ${ }^{10,11,13}$ Our patient experienced persistent nasal obstruction with epiphora and recurrent right conjunctivitis (due to the $a b$ extrinseco compression of the nasolacrimal duct), repeated and self-limiting epistaxis, and a slight deformation of the nasal pyramid.

The differential diagnoses included an ameloblastoma, giant-cell granuloma, ossifying fibroma, epithelial cyst or sarcoma, vascular lesions, malignant carcinoma, rhabdomyosarcoma, and teratoma. ${ }^{3,14,15}$ In the case of nasal involvement, further etiologies that should be considered are dermoid cysts, congenital mid-line malformations, and inflammatory polyps secondary to an intranasal foreign body. Vascular lesions should be investigated by means of dedicated second- and third-level imaging studies before undertaking any bioptic or surgical procedure in order to avoid potentially severe bleeding.

The diagnosis of an ABC is mainly based on typical radiological imaging and pathological findings. ${ }^{10-12} \mathrm{CT}$ scans show ABCs involving the long bones as lesions with regular and well-defined margins surrounded by osteolytic resorption; in the spinal cord, they are associated with pronounced para-osseous tumour expansion without osteolysis, and well-defined margins surrounded by structural vescicular lacunae. ${ }^{16}$ The CT characteristics of previously described ENT locations show local expansion of the mass associated with bone resorption as a sign of lytic activity, and the presence of multiple fluid-containing cavities separated by interior septa. ${ }^{10}$

Complementary MRI is required to evaluate soft tissue involvement especially in the case of central nervous system involvement and, in the case of spinal cord lesions, to detect neural tissue compression. In other locations, CT angiography could be considered the better diagnostic tool.

MRI findings include one or more blood-filled cysts with contrast-enhancing stroma, and the frequent presence of fluid. Signal intensity in T1- and T2-weighted images varies because of the different oxidation levels of blood and blood breakdown products in cyst fluid. ${ }^{17}$ In our patient, MRI confirmed the presence of a multilocular vascularised mass indicative of an $\mathrm{ABC}$.

In the case of large cysts, angiography is required to determine whether arterial embolization should be considered before surgery in order to reduce the risk of severe intra-operative bleeding ${ }^{16}$, but we also used it to exclude the hypothesis of vascular malformations or a juvenile nasopharyngeal angiofibroma. Moreover, nasal endoscopy was used as a firstline assessment in order to explore the nasal cavities and define their mucosal characteristics. Histopathology can confirm the diagnosis by showing a number of blood-filled cavernous or sinusoidal spaces adjacent to fibrous connective tissue stroma consisting of multinucleated osteoclasts similar to giant-cells. ${ }^{18}$

$\mathrm{ABCs}$ generally require a surgical approach, such as curettage, piecemeal resection or complete removal; however, as there is a $30 \%$ rate of recurrence after non-radical surgery, complete surgical excision is strongly recommended. ${ }^{19}$ We used minimally invasive endoscopic piecemeal resection extended to the right inferior turbinate and the outcome was good.

ABCs are radio-sensitive tumors, and some authors have described the use of radiotherapy alone or adjuvant radiotherapy; however, this treatment can lead to the cyst becoming a sarcoma. ${ }^{20}$ We did not consider radiotherapy because of our patient's age, and the location and limited size of the cyst.

ABCs rarely involve the nasal cavity but, when they do, they present with nasal obstruction and epistaxis. An endoscopic evaluation and dedicated second- and third-level imaging techniques should be used in the diagnostic work-up in order to confirm the diagnosis and exclude other conditions. Complete resection 
by means of a minimally invasive intranasal endoscopy is the treatment of choice.

\section{REFERENCES}

1. Van Arsdale WW. II. Ossifying haematoma. Ann Surg 1893; 18: 8-17.

2. Jaffè HL, Lichtenstein L. Solitary unicameral bone cyst. With emphasis on roentgen picture, the pathological appearance and the pathogenesis. Arch Surg 1942; 44: 1004-1025.

3. Martinez V, Sissons HA. Aneurysmal bone cyst. A review of 123 cases including primary lesions and those secondary to other bone pathology. Cancer 1988; 61: 2291-2304.

4. Perrotti V, Rubini C, Fioroni M, Piattelli A. Solid aneurysmal bone cyst of the mandible. Int J Pediatr Otorhinolaryngol 2004; 68: 1339-1344.

5. Bernier JL, Bhaskar SN. Aneurysmal bone cyst of the mandible. Oral Surg Oral Med Oral Pathol 1958; 11: 1018-1028.

6. Johnson TE, Bergin DJ, McCord CD. Aneurysmal bone cyst of the orbit. Ophthalmology 1988; 95: 86-89.

7. Hrishhikesh KA, Narlawar RS, Deasi SB, Aniruddha $\mathrm{K}$, Maheshwari S. Case report: Aneurysmal bone cyst of the ethmoid bone. Br J Radiol 2002; 75: 916-918.

8. Cansiz H, Inci E, Erisir F, et al. Aneurysmal bone cyst of the sphenoid bone. J Craniofaciac Surg 2002; 13: 411-414.

9. Motamedi $\mathrm{MH}$, Khodayari A. Aneurysmal bone cyst mimicking a malignancy. J Oral Maxillofac Surg 1993; 51: 691-695.

10. Guilemany JM, Alobid I, Blanch JL, Ballesteros F, Alós L, Mullol J. Orbitoethmoid aneurysmal bone cyst. Case report and literature review. Rhinology 2004; 42: $164-166$
11. Ruiz de la Cuesta F, Alenda C, Monerris E, Gras Albert JR. Aneurysmal bone cyst of the ethmoid: Apropos of a case and review of the medical literature. Acta Otorrinolaringol Esp 2007; 58: 113-115.

12. Simsek G, Saka C, Sonbay DN, Akin I, Koybasioglu F. Aneurysmal bone cyst in the middle turbinate: A case report. Ear Nose Throat J 2013; 9: E47.

13. Winnepenninckx V, Debiec-Rychter M, Jorissen $M$, Bogaerts S, Sciot R. Aneurysmal bone cyst of the nose with 17p13 involvement. Virchows Arch 2001; 439: 636-639.

14. Strabbing EM, Gortzak RA, Vinke JG, Saridin CP, van Merkesteyn JP. An atypical presentation of a solitary bone cyst of the mandibular ramus: A case report. J Craniomaxillofac Surg 2011; 39: 145-147.

15. Sciot R, Dorfman H, Brys P, et al. Cytogeneticmorphologic correlations in aneurysmal bone cyst, giant cell tumor of bone and combined lesions. A report from the CHAMP study group. Mod Pathol 2000; 13: 1206-1210.

16. Meyer S, Reinhard H, Graf N, Kramann B, Schneider G. Arterial embolization of a secondary aneurysmatic bone cyst of the thoracic spine prior to surgical excision in a 15-year-old girl. Eur J Radiol 2002; 43: 79-81.

17. Beltran J, Simon DC, Levy M, Herman L, Weis L, Mueller CF. Aneurysmal bone cysts: MR imaging at 1.5 T. Radiology 1986; 158: 689-690.

18. Rosai J. Bones. In: Rosai J, Ackerman LV, (eds). Ackerman's surgical pathology. (9th ed). St. Louis: CV Mosby; 2004: 2137.

19. Zielnik B, Gutkowska J. A case of aneurysmal cyst of the ethmoid sinus. Otolaryngol Pol 1995; 49: 169-172.

20. Matt BH. Aneurysmal bone cyst of the maxilla: Case report and review of the literature. Int J Pediatr Otorhinolaryngol 1993; 25: 217-226. 